\title{
Scoping Review of Neuroimaging Studies Investigating Frailty and Frailty Components
}

\author{
David López-Sanz ${ }^{1,2 \dagger}$, Isabel Suárez-Méndez ${ }^{1 \dagger}$, Raquel Bernabé ${ }^{3}$, Natalia Pasquín ${ }^{3}$, \\ Leocadio Rodríguez-Mañas ${ }^{3,4,5}$, Fernando Maestú ${ }^{1,2,6}$ and Stefan Walter ${ }^{3,5,7 *}$ \\ ${ }^{1}$ Laboratory of Cognitive and Computational Neuroscience (UCM-UPM), Centre for Biomedical Technology (CTB), Technical \\ University of Madrid (UPM), Madrid, Spain, ${ }^{2}$ Department of Experimental Psychology, Complutense University of Madrid \\ (UCM), Madrid, Spain, ${ }^{3}$ Fundación Para la Investigación Biomédica, Getafe University Hospital, Madrid, Spain, ${ }^{4}$ Geriatrics \\ Department, Getafe University Hospital, Madrid, Spain, ${ }^{5}$ Centro de Investigación Biomédica en Red Fragilidad y \\ Envejecimiento Saludable (CIBERFES), Madrid, Spain, ${ }^{6}$ Centro de Investigación Biomédica en Red en Bioingeniería, \\ Biomateriales y Nanomedicina (CIBER-BBN), Zaragoza, Spain, ${ }^{7}$ Department of Epidemiology and Biostatistics, University of \\ California, San Francisco, San Francisco, CA, United States
}

OPEN ACCESS

Edited by:

Gil Atzmon,

University of Haifa, Israel

Reviewed by:

Wee Shiong Lim,

Tan Tock Seng Hospital, Singapore Renato Gorga Bandeira De Mello, Universidade Federal do Rio Grande do Sul (UFRGS), Brazil

*Correspondence:

Stefan Walter

swalter@psg.ucsf.edu

tThese authors have contributed equally to this work and shared first authorship

Specialty section:

This article was submitted to Geriatric Medicine

a section of the journal

Frontiers in Medicine

Received: 09 July 2018 Accepted: 17 September 2018 Published: 08 October 2018

Citation:

López-Sanz D, Suárez-Méndez I, Bernabé R, Pasquín N, Rodríguez-Mañas L, Maestú $F$ and Walter S (2018) Scoping Review of Neuroimaging Studies Investigating

Frailty and Frailty Components.

Front. Med. 5:284.

doi: 10.3389/fmed.2018.00284
Background: Neuroimaging techniques are a cornerstone for diagnosing and investigating cognitive decline and dementia in the elderly. In frailty research, the physical as opposed to the cognitive domain of the aging process, neuroimaging studies are less common. Here we systematically review the use of neuroimaging techniques in frailty research.

Methods: We searched PUBMED for any publication reporting the association between neuroimaging markers and frailty, following Fried's original definition, as well as its determining phenotypes: gait speed, grip strength, fatigue and recent weight loss in the non-diseased population older than 65 years.

Results: The search returned a total of 979 abstracts which were independently screened by 3 reviewers. In total, 17 studies met the inclusion criteria. Of these, 12 studies evaluated gait speed, 2 grip strength, and 3 frailty (2 Fried Frailty, 1 Frailty Index). An association between increased burden of white matter lesions, lower fractional anisotropy, and higher diffusivity has been associated consistently to frailty and worse performance in the different frailty components.

Conclusions: White matter lesions were significantly associated to frailty and frailty components thus highlighting the potential utility of neuroimaging in unraveling the underlying mechanisms of this state. However, considering small sample size and design effects, it is not possible to completely rule out reverse causality between frailty and neuroimaging findings. More studies are needed to clarify this important clinical question.

Keywords: frailty, neuroimaging (anatomic and functional), review, gait speed, grip strength

\section{INTRODUCTION}

The number of older people in the global population is rapidly growing. From 2013 to 2060 the percentage of the population aged over 65 years is projected to increase from 18 to $28 \%$ and the proportion of those aged over 80 years will rise from 5 to $12 \%$ (1). Increased longevity raises social and economic challenges and has deep implications for the planning and delivery 
of healthcare. Indeed, as the number of older people rises, so does the number of people with age-related disability and dependence that require support with daily activities, healthcare services and/or institutionalization.

The transition from a robust status to one of age-related disability is usually preceded by a physiological state termed frailty $(2,3)$. Although frailty can be characterized using classical clinical phenotypes and laboratory-based biomarkers, a universally accepted definition of frailty remains to be agreed upon $(4,5)$. The most widely accepted definition of frailty is "an age-associated biological syndrome, characterized by a decrease of the biological reserve and resistance to stress due to a decline in several physiological systems. This places the individual in a special risk category when facing minor stressors and is associated with poor outcomes (disability, hospitalization and death)" (6). The most prominent approach used to assess frailty is using Fried's Frailty Criteria (7). Following this model, frailty is diagnosed based on the presence of at least three of the five physical attributes and capabilities of an individual. These include: weight loss (unintentional weight loss of $4.5 \mathrm{~kg}$ or more in the last year), exhaustion (self-reported), physical inactivity, slow walking speed, and weakness (low grip strength).

Many research initiatives, including the large scale European FRAILOMIC initiative, investigate OMIC factors associated to frailty $(4,8)$. In a recent seminar published in the Lancet (6), the authors discuss under the subheading «The Frail Brain》 only the structural and physiological changes taking place in the brain that are known to be associated with chronological age but not with frailty specifically. They reference the relationship between frailty and cognition as an example of the frail brain rather than answering which specific structural and physiological changes in the brain are associated with frailty.

In this scoping review the objective is to summarize the use of neuroimaging techniques in investigating Fried Frailty in the non-diseased, elderly population. In addition, we want to narratively outline whether current knowledge supports an overlap with dementia research.

\section{METHODS}

Following PRISMA methodology, for this scoping review we searched PubMed looking for works published prior to February 2018 (9-11).

We used the following query:

Neuroimaging [MESH] AND (Frailty OR (gait velocity OR gait speed) OR (grip strength OR muscle strength) OR fatigue OR weight loss)

We restricted the result set to those investigating humans using the PubMed filter functionality and adults older than 65 years. Nine Hundred and Seventy-Nine abstracts were reviewed independently by three researchers (SW, RB, and NP) with the help of abstrackr software without using the prediction algorithm (12). We excluded 958 papers, including those that investigated the relationship between neuroimaging markers and frailty parameters such as gait speed or grip strength only in diseased populations (e.g., Parkinson's Disease, Stroke, etc.) after reviewing the abstract. Of the remaining 21 papers that passed through full text screening, 13 were excluded for different reasons: not investigating frailty or its components $(n=6)$, study design not restricted to a population of 65 years of age or older $(n=5)$, inadequate study design $(n=1)$ and not including a neuroimaging marker $(n=1)$. When reviewing the references from the 21 articles originally deemed eligible after abstract screening, 9 studies were further considered eligible (Figure 1).

\section{RESULTS}

Of the 17 studies that fulfilled the inclusion criteria (Table 1), 3 studied frailty (2 Fried Frailty, 1 Frailty Index), 2 studies investigated grip strength, and 12 studies investigated gait speed or gait parameters. All but three studies were cross-sectional in nature. Table 2 lists the details of the outcome assessment, the imaging risk factors studied, the application of confounder control, and the conclusions for each of the studies included in this review.

In total, a maximum of 7,026 independent individuals participated in the studies evaluated, with the median study size

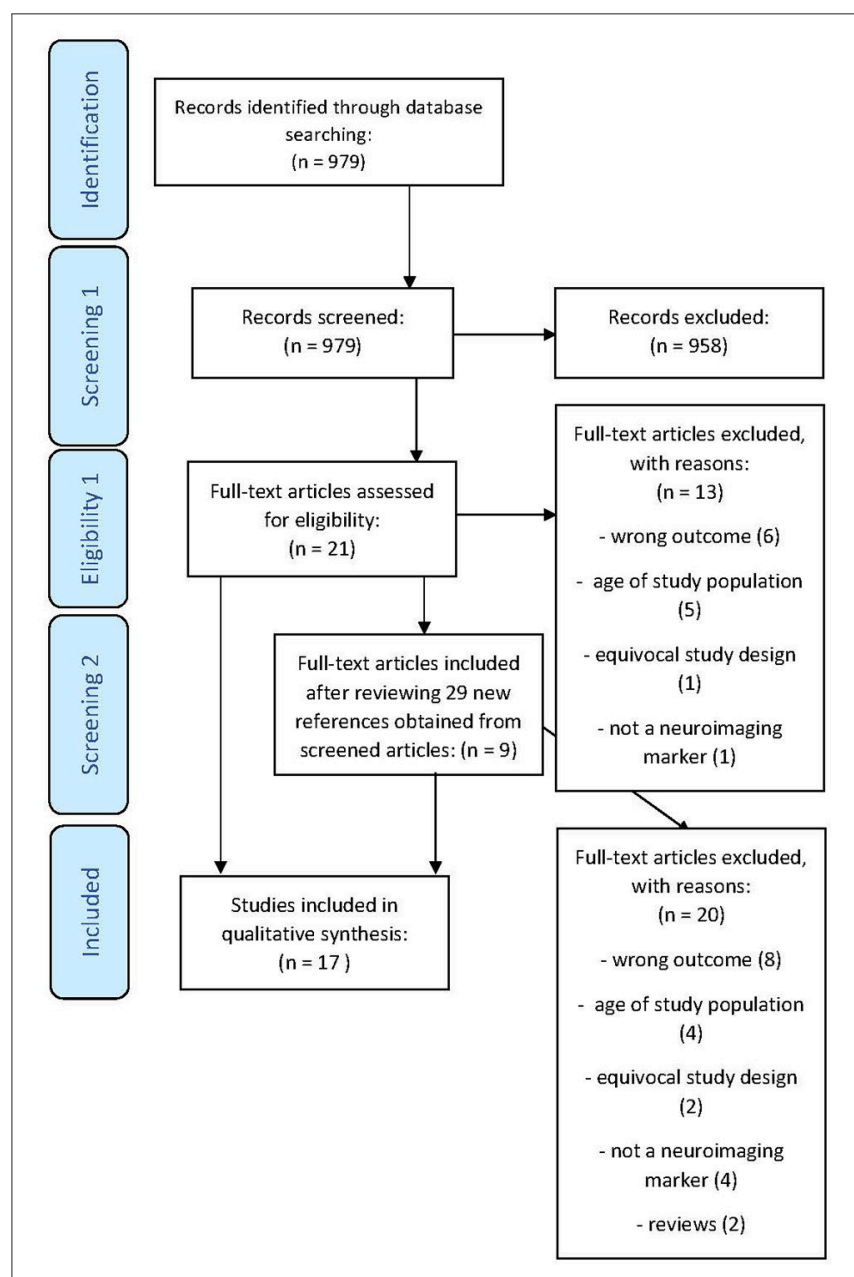

FIGURE 1 | PRISMA flow diagram. 
TABLE 1 | Descriptive overview of reviewed studies.

\begin{tabular}{|c|c|c|c|c|c|c|}
\hline References & Year & $\mathbf{N}(\% \text { female })^{\star}$ & Age (mean, SD) & Design & Outcome & Imaging technique \\
\hline \multicolumn{7}{|l|}{ FRAILTY } \\
\hline (13) & 2017 & $176(40.0 \%)$ & $75.0(5.2)$ & Cross-sectional & Frailty & Structural (T1-weighted MRI incl. DTI) \\
\hline$(14)$ & 2014 & $87(62.1 \%)$ & $\begin{array}{c}\text { Median } 78(\mathrm{IQR} \\
74-83)\end{array}$ & & Frailty & Structural (T2-weighted MRI) \\
\hline$(15)$ & 2001 & 4735 (42.8\%) & $72.7(4.33)$ & Cross-sectional & Frailty & Structural MRI (Image weighting not specified) \\
\hline \multicolumn{7}{|c|}{ GRIP STRENGTH } \\
\hline$(16)$ & 2015 & $191(53.4 \%)$ & $70.3(4.8)$ & Cross-sectional & Grip Strength & Functional MRI (Resting State) \\
\hline$(17)$ & 2016 & $165(51 \%)$ & $70.15(4.50)$ & Cross-sectional & Grip Strength & $\begin{array}{l}\text { Structural (T1-weighted MRI incl. DTI) and } \\
\text { Functional (Resting State) }\end{array}$ \\
\hline \multicolumn{7}{|l|}{ GAIT SPEED } \\
\hline (18) & 2010 & $148(56.1 \%)$ & 79 (IQR 76 - 83) & Cross-sectional & Gait Speed & Structural (T1-weighted MRI) \\
\hline (19) & 2012 & $214(64.5 \%)$ & $72.82(3.77)$ & Cross-sectional & Gait Speed & Structural (T1-weighted MRI) \\
\hline$(20)$ & 2010 & 795 (58.9\%) & $75.6(5.5)$ & Cross-sectional & Gait Speed & Structural (T1-weighted MRI) \\
\hline$(21)$ & 2016 & $265(57 \%)$ & $82.9(2.7)$ & Cross-sectional & Gait Speed & Structural (T2-weighted MRI incl. DTI) \\
\hline (22) & 2015 & $30(55.17 \%)$ & $72.5(5.22)$ & Cross-sectional & Gait Speed & Functional MRI (Resting State) \\
\hline (23) & 2008 & $104(61.5 \%)$ & $85.1(5.6)$ & Longitudinal & Gait Speed & Structural MRI (T1 and T2-weighted) \\
\hline (24) & 2009 & $1702(60.6 \%)$ & $72.4(4.1)$ & Longitudinal & Gait Speed & Structural MRI (T1 and T2-weighted) \\
\hline (25) & 2007 & 327 (56.5\%) & $78.2(3.9)$ & Cross-sectional & Gait Speed & Structural (T1 and T2-weighted MRI) \\
\hline$(26)$ & 2005 & $2450(57 \%)$ & $74.4(4.7)$ & Longitudinal & Gait Speed & Structural (T1 and T2-weighted MRI) \\
\hline$(27)$ & 1999 & $50(62 \%)$ & $85.1(7.2)$ & Cross-sectional & Gait Speed & Structural (T1-weighted MRI) \\
\hline$(28)$ & 2003 & 97 (40.2\%) & $78-79$ & Cross-sectional & Gait Speed & Structural (T2-weighted MRI) \\
\hline (29) & 2000 & $390(0 \%)$ & $72.37(2.96)$ & Cross-sectional & Gait Speed & Structural MRI (Image weighting not specified) \\
\hline
\end{tabular}

"The $n$ refers to the source population for which the descriptive statistics \% female and mean age are reported.

being of 191 participants, ranging from as low as $n=30$ for the functional MRI study of gait speed (22) to $n=2,450$ for the study with participants from the Cardiovascular Health Study (CHS) (26) published in 2005. None of the studies estimated a population effect by reweighting to the source or even general population (30).

\section{Findings by Frailty and Frailty Components Frailty}

In total, 3 studies were identified that investigated the association of structural brain parameters with frailty using MRI $(13,15,31)$. Two of these, Avila-Funes et al. and Newman et al., analyzed directly the frailty phenotype originally proposed by Linda Fried (7), and Jung et al. reported in a letter the association between white matter abnormalities and a Frailty Index conceptualized as a combination of basic and instrumental activities of daily living, physical performance, cognitive function and serum albumin level. This index showed a significant correlation (Spearman's $=0.49, p<0.001$ ) with Fried Frailty (14). All studies conclude that a higher burden of White Matter Lesions (WML) volume was associated with the prevalence of frailty. In addition, the original study from Newman et al. in participants from the above-mentioned CHS found evidence for a higher number of infarct lesions and increased ventricular size in frail participants but no association with sulcus size. Furthermore, Avile-Funes et al. found that white matter integrity assessed using diffusion tensor imaging was less preserved in frail participants from the AMIage study (13). This study investigated the relationship between fractional anisotropy (FA, lower in frail vs. non-frail participants), axial diffusivity ( $\mathrm{AD}$, higher), radial diffusivity ( $\mathrm{RD}$, higher), and mean diffusivity (MD, higher) across white matter tracts including the corpus callosum, anterior limb of internal capsule, external capsule, and posterior thalamic radiations.

All these studies adjusted for major confounders such as age, gender, and major age-associated diseases and were nested in longitudinal cohort studies.

\section{Grip Strength}

Hirsiger et al. evaluated the association between grip strength and structural/functional connectivity in the cingulum during resting state as obtained from DTI and fMRI respectively in 165, cognitively normal older participants (mean age 70.15) from the longitudinal healthy aging brain (LHAB) project of the University of Zurich, Switzerland (17). They found that an increase in FA in the cingulum bundle was positively associated with grip strength $(p=0.022)$ while an increase in mean diffusivity was negatively associated with grip strength $(p=0.018)$ in models adjusted for age, gender, education, and diastolic blood pressure. Resting state functional connectivity in the cingulum, more concretely the correlation between posterior cingulate cortex and medial prefrontal cortex BOLD signals, was not associated to grip strength $(p=0.270)$.

Seidler et al. evaluated the same study sample as Hirsiger et al. but looking at individual regions-of-interest (ROIs), using left primary motor cortex, left putamen and right cerebellum lobules $\mathrm{V}$ and VIII, all of them associated to hand motor performance 
TABLE 2 | Outcome, Imaging Risk Factors, and Conclusions from reviewed studies.

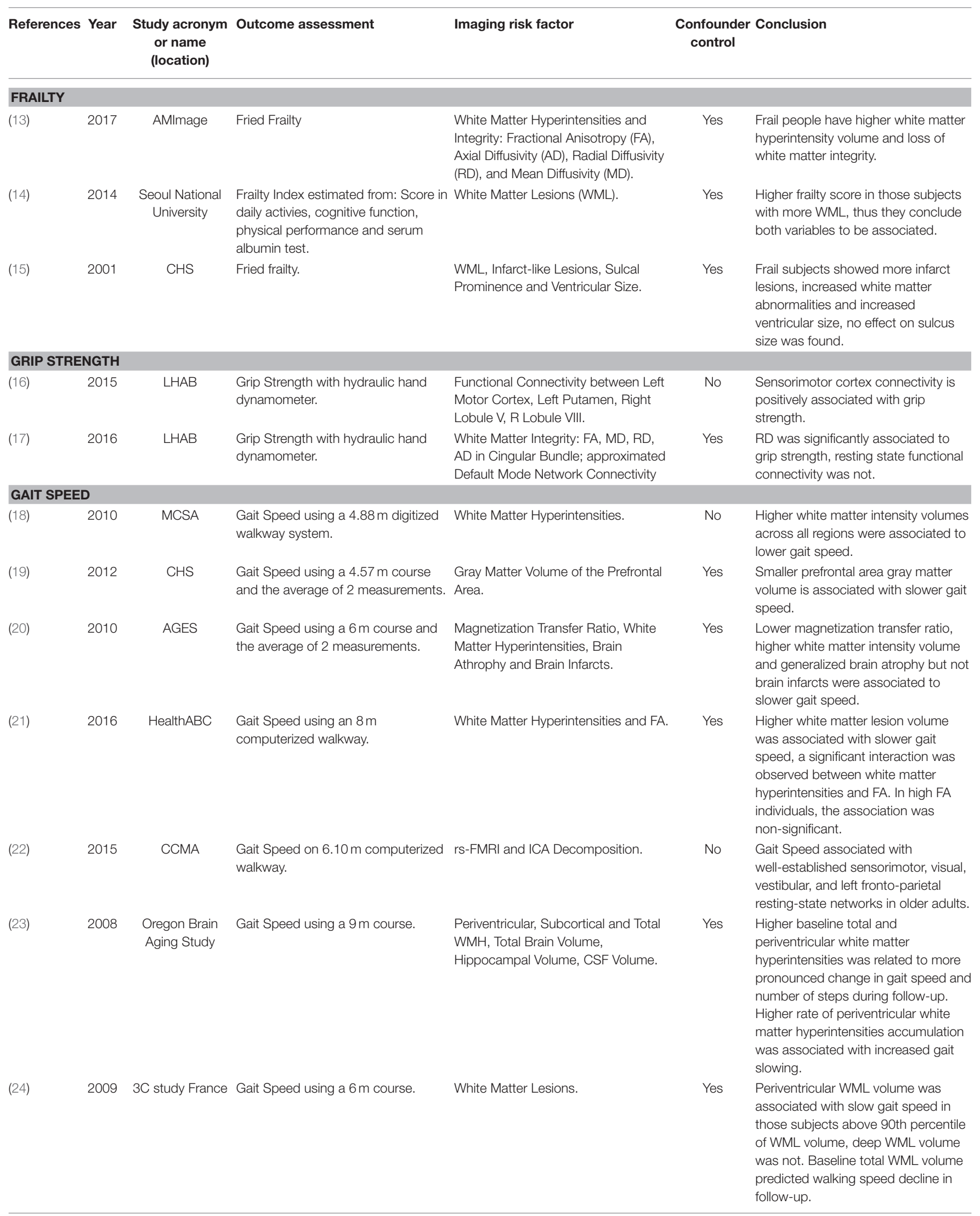


TABLE 2 | Continued

\begin{tabular}{|c|c|c|c|c|c|c|}
\hline References & Year & $\begin{array}{l}\text { Study acronym } \\
\text { or name } \\
\text { (location) }\end{array}$ & Outcome assessment & Imaging risk factor & $\begin{array}{l}\text { Confounder } \\
\text { control }\end{array}$ & Conclusion \\
\hline (25) & 2007 & $\mathrm{CHS}$ & $\begin{array}{l}\text { Gait Speed using a } 4.57 \mathrm{~m} \text { course } \\
\text { and Balance checking the ability to } \\
\text { hold semitandem position for at least } \\
10 \mathrm{~s} .\end{array}$ & $\begin{array}{l}\text { Gray Matter Volume of ROls known to } \\
\text { be associated with mobility. }\end{array}$ & Yes & $\begin{array}{l}\text { Smaller gray matter volumes } \\
\text { remained associated with slow gait } \\
\text { and poor balance after cofounder } \\
\text { control in LH smaller cerebellum and } \\
\text { dorsolateral prefrontal regions (slower } \\
\text { gait) and RH basal ganglia, superior } \\
\text { posterior parietal cortex and } \\
\text { cerebellum (balance difficulty). }\end{array}$ \\
\hline (26) & 2005 & $\mathrm{CHS}$ & Gait Speed using a $4.57 \mathrm{~m}$ course. & $\begin{array}{l}\text { Ventricular Enlargement, White Matter } \\
\text { Hyperintensities, Subcortical and } \\
\text { Basal Ganglia Small Brain Infarcts. }\end{array}$ & Yes & $\begin{array}{l}\text { Presence of structural brain } \\
\text { abnormalities was associated with } \\
\text { greater risk of incident functional } \\
\text { impairment and greater risk of gait } \\
\text { speed decline after cofounder control. }\end{array}$ \\
\hline (27) & 1999 & $\begin{array}{l}\text { Oregon Brain } \\
\text { Aging Study }\end{array}$ & Gait Speed using a 9.14 m course. & $\begin{array}{l}\text { Total Brain Volume, Intracranial } \\
\text { Volume, Ventricular Volume, } \\
\text { Periventricular High Signal, Deep High } \\
\text { Signal. }\end{array}$ & Yes & $\begin{array}{l}\text { Ventricular volume and periventricular } \\
\text { white matter high signal volume, but } \\
\text { not total brain volume or deep white } \\
\text { matter high signal, were correlated } \\
\text { gait speed independent of age. }\end{array}$ \\
\hline (28) & 2003 & ABC 1921 Study & Gait Speed using a 6 m course. & $\begin{array}{l}\text { WML, Periventricular Lesions and } \\
\text { Brain Stem Lesions. }\end{array}$ & No & $\begin{array}{l}\text { Decreased gait speed correlated } \\
\text { significative with an increased grade } \\
\text { of brain stem lesions. }\end{array}$ \\
\hline (29) & 2000 & NHLBI Twin Study & $\begin{array}{l}\text { Gait Speed using a } 2.43 \text { m course } \\
\text { (faster of two walks). }\end{array}$ & $\begin{array}{l}\text { White Matter Hyperintensities, Total } \\
\text { Cranial Brain Volume (TCB). }\end{array}$ & Yes & $\begin{array}{l}\text { Above the median total brain volume } \\
\text { but not white matter hyperintensity } \\
\text { volume was associated with higher } \\
\text { gait speed. }\end{array}$ \\
\hline
\end{tabular}

(16). They found that resting state connectivity between the motor cortex, bilateral sensorimotor cortex and supplementary motor area was greater in participants with higher grip strength. They also found stronger connectivity between the putamen region, medial frontal cortex and precuneus, as well as between the cerebellar seeds, the frontal cortex and temporal regions associated with higher grip strength. In addition, cerebellar lobule $\mathrm{V}$ showed increased connectivity with lobules VIIIa and VIIIb with greater grip strength.

\section{Gait Speed}

Twelve studies using data from 9 population studies investigated gait speed. 11 studies used structural MRI imaging for testing, among other aspects, the association between WML $(n=9)$ $(18,20,21,23,24,26-29)$ and gray matter $(n=2)(19,25)$ with gait speed, while one study evaluated resting state networks using fMRI and their association with gait speed (22).

In each study relating gait speed to neuroimaging markers, gait speed was assessed differently. Nevertheless, all but one study used velocity in units of distance $(\mathrm{m}$ or $\mathrm{cm})$ per second as outcome measure rather than time in seconds for walking a predefined distance.

Nine studies investigated the relationship between gait speed and WML. Generalized measures of WML were associated with slower gait in models adjusted for major confounders in eight out of nine studies. Only the NHLBI Twin Study, one of the earliest neuroimaging studies from the year 2000, did not report a significant effect, although the tendency was consistent with the other works. Some studies $(23,24$, 27) also analyzed the effect of periventricular WML burden coming to the same conclusions. In these studies, deep WML volume was not associated with gait speed. In addition, Silbert et al. examined the effect of change in WML volume and concluded that the accumulation of WML was associated with increased gait slowing during follow-up. Rosario et al. additionally investigated the possibility of an interaction effect between WML and white matter integrity measured by FA in participants from The Health, Aging and Body Composition Study (Health $A B C$ ) and found that the association between WML volume and gait speed was not significant in high FA individuals.

Two studies by Rosano et al. $(19,20)$ in participants from the CHS and Age, Gene/Environment Susceptibility (AGES) study investigated the association between gray matter volume and gait speed. Using a ROIs approach of areas a priori known to be associated to mobility, they identified an association between small volumes in cerebellum and dorsolateral prefrontal regions (25) and prefrontal gray matter volume (19) with slower gait. In addition, brain atrophy-defined by an atrophy index computed as (intracranial volume-brain volume)/intracranial volume-but not cerebral infarcts were associated with reduced gait speed in the AGES study.

The only study investigating resting state connectivity via functional MRI in participants from the Central Control of Mobility in Aging (CCMA) study confirmed an association between well-established sensorimotor, visual, vestibular, and left 
fronto-parietal resting state networks and gait speed in older adults.

\section{DISCUSSION}

Neuroimaging techniques and in particular functional neuroimaging, a cornerstone for diagnosing and investigating cognitive decline and dementia in the elderly, are hardly used to identify biomarkers and risk factors associated to frailty. This is surprising given the close link between frailty and cognitive decline which has led to «cognitive frailty $\gg$ becoming a major research topic (32-34). As of end of January 2018, only 3 studies directly assessed the association between frailty and neuroimaging markers identifying a relationship between an increased burden of white matter hyperintensities, lower fractional anisotropy and higher diffusivity with a higher prevalence of frailty. None of these studies evaluated connectivity or any other functional metric. Furthermore, among these studies, the different frailty components have received uneven attention with many more studies focusing in the relationship between neuroimaging markers and gait speed compared to handgrip strength. A higher burden of white matter hyperintensities has been associated to lower gait speed. Furthermore, lower fractional anisotropy and an increase in mean diffusivity were associated to low gait speed and grip strength.

More white matter hyperintensities and lower white matter structural integrity were found to be associated with an increased prevalence of frailty, lower grip strength and slower gait in all studies that investigated this neuroimaging risk factor and were considered for this review. These results support investigative efforts into the role of the central nervous system and vascular damage as possibly being implicated in the pathophysiology of frailty. Findings supporting these results highlight the association between structural changes and WML with physical fitness and activity (35). In fact, white matter hyperintensities, possibly the result of arteriosclerotic processes, are almost ubiquitous in the elderly (36) and their presence is facilitated by the exposure in mid-life to well-known risk factors such as smoking, hypertension, diabetes mellitus, and chronological age (37). They are also consistently associated with cognitive impairment (37). However, to be associated with global cognitive decline, the presence of other lesions is required and by themselves they cannot be used as an indicator of dementia (38). As such, it is problematic to infer the role of WML in the development of frailty from the knowledge available to date, particularly since most of the studies reviewed here and all that directly investigated the frailty phenotype are cross-sectional and the reported findings could be a result of reverse causation. Accumulating longitudinal evidence in the fields of stroke, dementia and mortality, supports the role of white matter hyperintensities as a risk factor for these endpoints. But the associations reported for frailty, whether causal or not, might not be sufficient to back the classification of WML as a risk factor useful in the diagnosis or prognosis of frailty. Whether or not WML can provide useful information in combination with other biomarkers from the brain or OMICs remains to be evaluated.

White matter microstructure has been associated to frailty and its defining phenotypes in this review. DTI has emerged as a technique allowing the study of white matter changes occurring at a microscopic level before its macroscopic manifestations are visible on a structural MRI (39). DTI seeks to evaluate the loss of white matter microstructure integrity by characterizing the degree of restriction to movement across different ellipsoid axis $(\mathrm{AD}, \mathrm{RD}, \mathrm{MD})$ as well as the relative degree of anisotropy in a region of interest indicative of a preferential diffusion path. DTI's sensitivity to subtle abnormalities has encouraged its application to the study of the aging brain under both healthy and pathological conditions, yet only two of the studies considered in this review deal directly with the microstructural alterationsas extracted from the exploration of DTI parameters-regarding frailty condition (13) or frailty-related components (17). The first study informed of a greater loss of WM integrity (lower FA and higher diffusivity values) in frail participants. Local decreases in FA have been also observed in normal aging-involving frontal WM and anterior cingulum-while DTI abnormalities found in participants undergoing cognitive decline (MCI) or neurodegenerative disease $(\mathrm{AD})$ are also significant in posterior regions signaling a loci of irregularities that could be related to an Alzheimer's disease type pathology [for a systematic review, see (40)]. One of the regions reported in Avila-Funes et al. to exhibit a lower FA in frail older adults is the anterior limb of the internal capsule. This region has been subjected to some discrepancy in the $\mathrm{MCI}$ and $\mathrm{AD}$ literature. Some authors do not find significant reductions in FA $(41,42)$ while others do (43). The later suggest that motor dysfunction is part of the incipient process of $\mathrm{AD}$ but as this is not often clinically supported is thought to represent an uncommon subgroup within $\mathrm{AD}$ patients (40) that could be related to those individuals manifesting both a cognitive decline and a frailty condition. The anterior limb of the internal capsule, pinpointed in the study of Avila-Funes et al., is involved in the connection of frontal regions with different brain regions. Interestingly, frontal structural disconnection has been linked to cognitive decline in older adults, which seems to support the link between frailty and cognition. In Hirsiger et al., reduced grip strength was associated to the loss of WM microstructural integrity in the cingulum, a region whose fibers have been reported to present a significant FA reduction in MCI and even more in $\mathrm{AD}$ (44).

Many of the studies covered in this work agreed on the finding that brain volume reductions - manifested as either ventricular volume increase $(15,26,27)$ or a diffuse reduction in total brain volume $(20,29)$-are associated to classical phenotypes of frailty. However, the specific cortical atrophy pattern associated to physical frailty is yet to be fully established as very little work has addressed this question. In this vein, two of the studies reported significant reductions in prefrontal volume linked to slower gait speed $(19,25)$, which could shed some light in this regard. Gray matter atrophy is a hallmark of dementia progression and is closely linked to cognitive dysfunction (45). Interestingly, Silbert et al. (23) failed to find any significant relationship between gait speed and hippocampal volume, which is one of the first 
structures showing volume reduction in Alzheimer's Disease dementia (46). Nevertheless, it is important to bear in mind that the specific pattern of gray matter atrophy is highly dependent on the dementia cause. Interestingly, the comorbidity between physical frailty and cognitive deterioration leading to dementia observed in epidemiological studies, seems to be supported by the fact that frailty has been consistently linked to gray matter atrophy in the few neuroimaging studies available to date, which is solidly known to be also a major risk factor for dementia development (47).

Functional connectivity estimates the reciprocal interactions between distant brain regions as a function of the statistical dependence between their respective activity time courses. Synchronous activity has been reported to be consistently associated to cognitive (48) and even motor performance (49). However, although its influence in cognitive deterioration and dementia is receiving increasing attention, its role has been very scarcely studied in the context of frailty. From the reviewed literature only three works reported functional connectivity metrics. Hirsiger et al. (17) failed to find any statistical relationship between posterior cingulate cortex-medial prefrontal cortex connectivity and grip strength. This particular link represents one of the major features of the default mode network (DMN), which is associated to internal processing states and is a critically associated to dementia progression (50). However, the other two studies employing FC metrics $(16,22)$ included a larger set of regions in their analyses obtaining in both cases similar results, highlighting a significant hyposynchronization affecting particularly sensorimotor areas and prefrontal regions. Although sensorimotor network is not one of the key networks in dementia progression, frontoparietal network disruption (as reported by Yuon et al.) has been extensively linked to cognitive deterioration, particularly in attention and executive functions. This particular pattern of alterations could underlie the observed relationship between frailty and dementia risk. In general, functional neuroimaging techniques, such as MEG, have shown great utility in detecting the initial stages of dementia and its associations with amyloidbeta [for a review see (51)], which could be an important factor in explaining the link between frailty and dementia.

\section{LIMITATIONS}

This scoping review has important limitations. First, the restrictions to the Fried phenotype and the non-diseased, elderly $(65+$ years $)$ population, might have significantly reduced the study base. However as frailty phenotype is both more prevalent

\section{REFERENCES}

1. World Health Organization. World Report on Ageing and Health. Geneva: World Health Organization (2015).

2. Angulo J, El Assar M, Rodriguez-Manas L. Frailty and sarcopenia as the basis for the phenotypic manifestation of chronic diseases in older adults. Mol Aspects Med. (2016) 50:1-32. doi: 10.1016/j.mam.2016. 06.001 and potentially impactful in the older population we focused our review in that specific segment of the population. Nonetheless, to the best of our knowledge, this is the first review addressing the use of neuroimaging markers in frailty research, thus making it important to focus on research that approaches frailty from a broad perspective and in the non-diseased population to avoid coming to conclusions biased by results from specific diseased groups. Furthermore, although frailty definitions different from the Fried phenotype model exist $(6,52)$, it is still the most commonly employed. Second, as most of the studies reviewed are cross-sectional, reverse causality cannot be excluded, and the results reported here should be considered as mere statistical associations. Third, as in all observational research, residual confounding that artificially creates a statistical association between neuroimaging markers and frailty due to a common, unknown factor cannot be excluded. Fourth, as this review was restricted to the general, non-diseased population, we did not include different studies pinpointing a link between frailty and beta-amyloid accumulation in AD-related regions in atrisk population $(53,54)$. However, these studies could also be considered a very promising direction for future research into the relationship between dementia or cognitive dysfunction and frailty.

In conclusion, current literature supports the association between increased burden of white matter lesions, lower fractional anisotropy, and higher diffusivity with frailty and an overall worse performance in the different frailty components (i.e., gait speed and handgrip strength). However, the overall study base contributing to these findings is very small, mostly cross-sectional and does not allow for generalizations. Representative, longitudinal neuroimaging studies, structural and functional, investigating frailty and the subgroup of people that exhibit frailty and cognitive decline as comorbidity are urgently needed to identify processes that are specific to frailty or common to both frailty and cognitive decline and dementia to facilitate the differential diagnosis in the clinical setting.

\section{AUTHOR CONTRIBUTIONS}

SW had the original idea of the manuscript, participated in the literature search and selection, and authored the initial draft of the manuscript. DL-S, IS-M, RB, NP participated in the literature review, the abstract screening, and the results extraction, and authored sections of the manuscript. FM, LR-M provided important intellectual input to the manuscript. All authors read, contributed, and approved the final version of the manuscript.
3. de Vries NM, Staal JB, van Ravensberg CD, Hobbelen JSM, Olde Rikkert MGM, Nijhuis-van der Sanden MWG. Outcome instruments to measure frailty: a systematic review. Ageing Res Rev. (2011) 10:104-14. doi: 10.1016/j.arr.2010.09.001

4. Erusalimsky JD, Grillari J, Grune T, Jansen-Duerr P, Lippi G, Sinclair AJ, et al. In search of 'Omics'-based biomarkers to predict risk of frailty and its consequences in older individuals: the FRAILOMIC initiative. Gerontology (2016) 62:182-90. doi: 10.1159/000435853 
5. Rodriguez-Manas L, Feart C, Mann G, Vina J, Chatterji S, ChodzkoZajko W, et al. Searching for an operational definition of frailty: a delphi method based consensus statement. the frailty operative definition-consensus conference project. J Gerontol Ser -Biol Sci Med Sci. (2013) 68:62-7. doi: 10.1093/gerona/gls119

6. Clegg A, Young J, Iliffe S, Rikkert MO, Rockwood K. Frailty in elderly people. Lancet (2013) 381:752-62. doi: 10.1016/S0140-6736(12)62167-9

7. Fried LP, Tangen CM, Walston J, Newman AB, Hirsch C, Gottdiener J, et al. Frailty in older adults: evidence for a phenotype. J Gerontol Ser A. (2001) 56:M146-57. doi: 10.1093/gerona/56.3.M146

8. Lippi G, Jansen-Duerr P, Vina J, Durrance-Bagale A, Abugessaisa I, Gomez-Cabrero D, et al. Laboratory biomarkers and frailty: presentation of the FRAILOMIC initiative. Clin Chem Lab Med. (2015) 53:E253-5. doi: 10.1515/cclm-2015-0147

9. Moher D. Preferred reporting items for systematic reviews and metaanalyses: the PRISMA statement. Ann Intern Med. (2009) 151:264-9. doi: 10.1371/journal.pmed.1000097

10. McGaghie WC. Varieties of integrative scholarship: why rules of evidence, criteria, and standards matter. Acad Med. (2015) 90:294-302. doi: 10.1097/ACM.0000000000000585

11. Arksey H, O'Malley L. Scoping studies: towards a methodological framework. Int J Soc Res Methodol. (2005) 8:19-32. doi: 10.1080/1364557032000119616

12. Rathbone J, Hoffmann T, Glasziou P. Faster title and abstract screening? Evaluating Abstrackr, a semi-automated online screening program for systematic reviewers. Syst Rev. (2015) 4:80. doi: 10.1186/s13643-015-0067-6

13. Avila-Funes JA, Pelletier A, Meillon C, Catheline G, Periot O, Trevin O-Frenk I, et al. Vascular cerebral damage in frail older adults: the AMImage Study. $J$ Gerontol A Biol Sci Med Sci. (2017) 72:971-7. doi: 10.1093/gerona/glw347

14. Jung H-W, Kim S-W, Ahn S, Lim J-Y, Han J-W, Kim T-H, et al. Prevalence and outcomes of frailty in korean elderly population: comparisons of a multidimensional frailty index with two phenotype models. PLoS ONE (2014) 9:e87958. doi: 10.1371/journal.pone.0087958

15. Newman AB, Gottdiener JS, McBurnie MA, Hirsch CH, Kop WJ, Tracy R, et al. Associations of subclinical cardiovascular disease with frailty. J Gerontol Ser A (2001) 56:M158-66. doi: 10.1093/gerona/56.3.M158

16. Seidler R, Erdeniz B, Koppelmans V, Hirsiger S, Mérillat S, Jäncke L. Associations between age, motor function, and resting state sensorimotor network connectivity in healthy older adults. Neuroimage (2015) 108:47-59. doi: 10.1016/j.neuroimage.2014.12.023

17. Hirsiger S, Koppelmans V, Mérillat S, Liem F, Erdeniz B, Seidler RD, et al. Structural and functional connectivity in healthy aging: associations for cognition and motor behavior: brain connectivity in aging. Hum Brain Mapp. (2016) 37:855-67. doi: 10.1002/hbm.23067

18. Murray ME, Senjem ML, Petersen RC, Hollman JH, Preboske GM, Weigand SD, et al. Functional impact of white matter hyperintensities in cognitively normal elderly subjects. Arch Neurol. (2010) 67:1379-85. doi: 10.1001/archneurol.2010.280

19. Rosano C, Studenski SA, Aizenstein HJ, Boudreau RM, Longstreth WT, Newman AB. Slower gait, slower information processing and smaller prefrontal area in older adults. Age Ageing (2012) 41:58-64. doi: 10.1093/ageing/afr113

20. Rosano C, Sigurdsson S, Siggeirsdottir K, Phillips CL, Garcia M, Jonsson PV, et al. Magnetization transfer imaging, white matter hyperintensities, brain atrophy and slower gait in older men and women. Neurobiol Aging (2010) 31:1197-204. doi: 10.1016/j.neurobiolaging.2008. 08.004

21. Rosario BL, Rosso AL, Aizenstein HJ, Harris T, Newman AB, Satterfield S, et al. Cerebral white matter and slow gait: contribution of hyperintensities and normal-appearing parenchyma. J Gerontol A Biol Sci Med Sci. (2016) 71:968-73. doi: 10.1093/gerona/glv224

22. Yuan J, Blumen HM, Verghese J, Holtzer R. Functional connectivity associated with gait velocity during walking and walking-while-talking in aging: a resting-state fMRI study: resting-state fMRI and GAIT. Hum Brain Mapp. (2015) 36:1484-93. doi: 10.1002/hbm.22717

23. Silbert LC, Nelson C, Howieson DB, Moore MM, Kaye JA. Impact of white matter hyperintensity volume progression on rate of cognitive and motor decline. Neurology (2008) 71:108-13. doi: 10.1212/01.wnl.0000316799.86917.37
24. Soumaré A, Elbaz A, Zhu Y, Maillard P, Crivello F, Tavernier B, et al. White matter lesions volume and motor performances in the elderly. Ann Neurol. (2009) 65:706-15. doi: 10.1002/ana.21674

25. Rosano C, Aizenstein HJ, Studenski S, Newman AB. A regions-of-interest volumetric analysis of mobility limitations in community-dwelling older adults. J Gerontol A Biol Sci Med Sci. (2007) 62:1048-55. doi: 10.1093/gerona/62.9.1048

26. Rosano C, Kuller LH, Chung H, Arnold AM, Longstreth WT, Newman AB. Subclinical brain magnetic resonance imaging abnormalities predict physical functional decline in high-functioning older adults. J Am Geriatr Soc. (2005) 53:649-54. doi: 10.1111/j.1532-5415.2005.53214.x

27. Camicioli R, Moore MM, Sexton G, Howieson DB, Kaye JA. Agerelated brain changes associated with motor function in healthy older people. J Am Geriatr Soc. (1999) 47:330-4. doi: 10.1111/j.1532-5415.1999. tb02997.x

28. Starr JM, Leaper SA, Murray AD, Lemmon HA, Staff RT, Deary IJ, et al. Brain white matter lesions detected by magnetic resonance [correction of resosnance] imaging are associated with balance and gait speed. J Neurol Neurosurg Psychiatry (2003) 74:94-8. doi: 10.1136/jnnp.74.1.94

29. Carmelli D, DeCarli C, Swan GE, Kelly-Hayes M, Wolf PA, Reed T, et al. The joint effect of apolipoprotein E epsilon 4 and MRI findings on lower-extremity function and decline in cognitive function. J Gerontol A Biol Sci Med Sci. (2000) 55:M103-9. doi: 10.1093/gerona/55.2.M103

30. LeWinn KZ, Sheridan MA, Keyes KM, Hamilton A, McLaughlin KA. Sample composition alters associations between age and brain structure. Nat Commun. (2017) 8:874. doi: 10.1038/s41467-017-00908-7

31. Jung H-W, Kim S-W, Yoon S-J, Choi J-Y, Kim K, Kim C-H. Associations between frailty, retinal microvascular changes, and cerebral white matter abnormalities in korean older adults. J Am Geriatr Soc. 62:2209-10. doi: 10.1111/jgs.13114

32. Buchman AS, Bennett DA. Cognitive frailty. J Nutr Health Aging (2013) 17:738-9. doi: 10.1007/s12603-013-0397-9

33. Godin J, Armstrong JJ, Rockwood K, Andrew MK. Dynamics of frailty and cognition after age 50: why it matters that cognitive decline is mostly seen in old age. J Alzheimers Dis. (2017) 58:231-42. doi: 10.3233/JAD-161280

34. Kelaiditi E, Cesari M, Canevelli M, van Kan GA, Ousset P-J, Gillette-Guyonnet S, et al. Cognitive frailty: rational and definition from an (I.A.N.A./I.A.G.G.) international consensus group. J Nutr Health Aging (2013) 17:726-34. doi: $10.1007 /$ s12603-013-0367-2

35. Sexton CE, Betts JF, Demnitz N, Dawes H, Ebmeier KP, Johansen-Berg H. A systematic review of MRI studies examining the relationship between physical fitness and activity and the white matter of the ageing brain. Neuroimage (2016) 131:81-90. doi: 10.1016/j.neuroimage.2015.09.071

36. de Leeuw F-E, de Groot JC, Achten E, Oudkerk M, Ramos L, Heijboer R, et al. Prevalence of cerebral white matter lesions in elderly people: a population based magnetic resonance imaging study. The Rotterdam Scan Study. J Neurol Neurosurg Psychiatry (2001) 70:9-14. doi: 10.1136/jnnp.70.1.9

37. Pantoni L, Poggesi A, Inzitari D. The relation between whitematter lesions and cognition. Curr Opin Neurol. (2007) 20:390-7. doi: 10.1097/WCO.0b013e328172d661

38. Frisoni GB, Galluzzi S, Pantoni L, Filippi M. The effect of white matter lesions on cognition in the elderly-small but detectable. Nat Rev Neurol. (2007) 3:620-7. doi: 10.1038/ncpneuro0638

39. Moseley M. Diffusion tensor imaging and aging - a review. NMR Biomed. (2002) 15:553-60. doi: 10.1002/nbm.785

40. Chua TC, Wen W, Slavin MJ, Sachdev PS. Diffusion tensor imaging in mild cognitive impairment and Alzheimer's disease: a review. Curr Opin Neurol. (2008) 21:83-92. doi: 10.1097/WCO.0b013e3282f4594b

41. Bozzali M, Falini A, Franceschi M, Cercignani M, Zuffi M, Scotti G, et al. White matter damage in Alzheimer's disease assessed in vivo using diffusion tensor magnetic resonance imaging. J Neurol Neurosurg Psychiatry (2002) 72:742-6. doi: 10.1136/jnnp.72.6.742

42. Takahashi S, Yonezawa H, Takahashi J, Kudo M, Inoue T, Tohgi H. Selective reduction of diffusion anisotropy in white matter of Alzheimer disease brains measured by 3.0 Tesla magnetic resonance imaging. Neurosci Lett. (2002) 332:45-8. doi: 10.1016/S0304-3940(02)00914-X

43. Medina D, DeToledo-Morrell L, Urresta F, Gabrieli JDE, Moseley M, Fleischman $\mathrm{D}$, et al. White matter changes in mild cognitive impairment 
and AD: a diffusion tensor imaging study. Neurobiol Aging (2006) 27:663-72. doi: 10.1016/j.neurobiolaging.2005.03.026

44. Zhang Y, Schuff N, Jahng G-H, Bayne W, Mori S, Schad L, et al. Diffusion tensor imaging of cingulum fibers in mild cognitive impairment and Alzheimer disease. Neurology (2007) 68:13-9. doi: 10.1212/01.wnl.0000250326.77323.01

45. Guo Y, Zhang Z, Zhou B, Wang P, Yao H, Yuan M, et al. Grey-matter volume as a potential feature for the classification of Alzheimer's disease and mild cognitive impairment: an exploratory study. Neurosci Bull. (2014) 30:477-89. doi: 10.1007/s12264-013-1432-x

46. Gordon BA, Blazey TM, Su Y, Hari-Raj A, Dincer A, Flores S, et al. Spatial patterns of neuroimaging biomarker change in individuals from families with autosomal dominant Alzheimer's disease: a longitudinal study. Lancet Neurol. (2018) 17:241-50. doi: 10.1016/S1474-4422(18) 30028-0

47. Meyer, J. S., Rauch, G. M., Rauch, R. A., Haque, A., and Crawford, K. (2000). Cardiovascular and other risk factors for Alzheimer's disease and vascular dementia. Ann. N. Y. Acad. Sci. 903, 411-423. doi: 10.1111/j.1749-6632.2000.tb06393.x

48. van den Heuvel MP, Hulshoff Pol HE. Exploring the brain network: a review on resting-state fMRI functional connectivity. Eur Neuropsychopharmacol. (2010) 20:519-34. doi: 10.1016/j.euroneuro.2010.03.008

49. Westlake KP, Nagarajan SS. Functional connectivity in relation to motor performance and recovery after stroke. Front Syst Neurosci. (2011) 5:8. doi: $10.3389 /$ fnsys. 2011.00008

50. Greicius MD, Srivastava G, Reiss AL, Menon V. Default-mode network activity distinguishes Alzheimer's disease from healthy aging: evidence from functional MRI. Proc Natl Acad Sci USA. (2004) 101:4637-42. doi: 10.1073/pnas.0308627101

51. López-Sanz D, Serrano N, Maestú F. The role of magnetoencephalography in the early stages of alzheimer's disease. Front Neurosci. (2018) 12:572. doi: $10.3389 /$ fnins. 2018.00572

52. Rockwood K, Mitnitski A. Frailty in relation to the accumulation of deficits. $J$ Gerontol A Biol Sci Med Sci. (2007) 62:722-7. doi: 10.1093/gerona/62.7.722

53. Yoon DH, Lee J-Y, Shin SA, Kim YK, Song W. Physical frailty and amyloid- $\beta$ deposits in the brains of older adults with cognitive frailty. J Clin Med. (2018) 7:169. doi: 10.3390/ jcm7070169

54. Hooper C, De Souto Barreto P, Coley N, Cesari M, Payoux P, Salabert AS, et al. Cross-sectional associations of fatigue with cerebral $\beta$-amyloid in older adults at risk of dementia. Front Med. (2017) 4:173. doi: 10.3389/fmed.2017. 00173

Conflict of Interest Statement: The authors declare that the research was conducted in the absence of any commercial or financial relationships that could be construed as a potential conflict of interest.

Copyright () 2018 López-Sanz, Suárez-Méndez, Bernabé, Pasquín, RodríguezMañas, Maestú and Walter. This is an open-access article distributed under the terms of the Creative Commons Attribution License (CC BY). The use, distribution or reproduction in other forums is permitted, provided the original author(s) and the copyright owner(s) are credited and that the original publication in this journal is cited, in accordance with accepted academic practice. No use, distribution or reproduction is permitted which does not comply with these terms. 